\title{
Liver transplantation as last-resort treatment for patients with bile duct injuries following cholecystectomy: a multicenter analysis
}

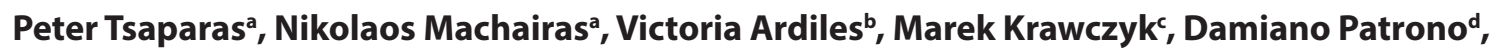 \\ Umberto Baccarani ${ }^{\mathrm{e}}$, Umberto Cillof, Einar Martin Aandahlg, Christian Cotsoglouh, \\ Johana Leiva Espinoza ${ }^{b}$, Rodrigo Sanchez Claríab, loannis D. Kostakis ${ }^{a}$, Aksel Foss ${ }^{g}$, \\ Vincenzo Mazzaferro' ${ }^{\mathrm{h}}$, Eduardo de Santibañes ${ }^{\mathrm{b}}$, Georgios C. Sotiropoulos ${ }^{\mathrm{a}, \mathrm{i}}$
}

Laiko General Hospital, National and Kapodistrian University of Athens, Athens, Greece; Hospital Italiano de Buenos Aires, Buenos Aires, Argentina; Medical University of Warsaw, Poland; University of Torino, Turin, Italy; University of Udine, Udine, Italy; University of Padova School of Medicine, Padova, Italy; Oslo University Hospital, Oslo, Norway; University of Milan, Milan, Italy; University Hospital Essen, Germany

\section{Abstract}

Background Liver transplantation (LT) has been used as a last resort in patients with end-stage liver disease due to bile duct injuries (BDI) following cholecystectomy. Our study aimed to identify and evaluate factors that cause or contribute to an extended liver disease that requires LT as ultimate solution, after BDI during cholecystectomy.

Methods Data from 8 high-volume LT centers relating to patients who underwent LT after suffering BDI during cholecystectomy were prospectively collected and retrospectively analyzed.

Results Thirty-four patients (16 men, 18 women) with a median age of 45 (range 22-69) years were included in this study. Thirty of them (88.2\%) underwent LT because of liver failure, most commonly as a result of secondary biliary cirrhosis. The median time interval between BDI and LT was 63 (range 0-336) months. There were 23 cases (67.6\%) of postoperative morbidity, 6 cases (17.6\%) of post-transplant 30-day mortality, and 10 deaths $(29.4 \%)$ in total after LT. There was a higher probability that patients with concomitant vascular injury (hazard ratio $10.69, \mathrm{P}=0.039$ ) would be referred sooner for LT. Overall survival following LT at 1, 3, 5 and 10 years was $82.4 \%$, $76.5 \%, 73.5 \%$ and $70.6 \%$, respectively.

Conclusion LT for selected patients with otherwise unmanageable BDI following cholecystectomy yields acceptable long-term outcomes.

Keywords Bile duct injury, cholecystectomy, liver transplantation, end-stage liver disease, cholangitis

Ann Gastroenterol 2020; 33 (6): 1-8

\section{Conflict of Interest: None}

Correspondence to: Nikolaos Machairas MD, PhD, $2^{\text {nd }}$ Department of Propaedeutic Surgery, General Hospital Laiko, Ag. Thoma 17, 11527 Athens, Greece, e-mail nmachair@gmail.com

Received 1 June 2020; accepted 20 July 2020; published online 2 October 2020

DOI: https://doi.org/10.20524/aog.2020.0541

${ }^{\text {a2 }}{ }^{\text {nd }}$ Department of Propaedeutic Surgery, Laiko General Hospital, National and Kapodistrian University of Athens, Athens, Greece (Peter Tsaparas, Nikolaos Machairas, Ioannis D. Kostakis, Georgios C. Sotiropoulos); ${ }^{b}$ Division of Hepatobiliary Surgery, Liver Transplant Unit, Hospital Italiano de Buenos Aires, Buenos Aires, Argentina
(Victoria Ardiles, Johana Leiva Espinoza, Rodrigo Sanchez Claría, Eduardo de Santibañes); 'Department of General, Transplant and Liver Surgery, Medical University of Warsaw, Poland (Marek Krawczyk); ${ }^{\mathrm{d}}$ General Surgery 2U, Liver Transplant Center, A.O.U. Città della Salute e della Scienza di Torino, University of Torino, Turin, Italy (Damiano Patrono); ${ }^{\mathrm{e}}$ Liver Transplant Unit, Department of Medicine, University

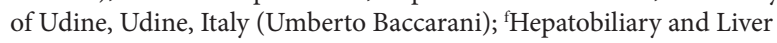
Transplant Unit, University of Padova School of Medicine, Padova,

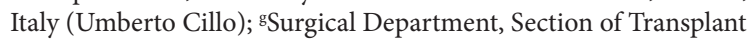
Surgery, Oslo University Hospital, Oslo, Norway (Einar Martin Aandahl, Aksel Foss); hepartment of Gastrointestinal Surgery and Liver Transplantation, Fondazione IRCCS Istituto Nazionale Tumori, University of Milan, Milan, Italy (Christian Cotsoglou); i Department of General Visceral and Transplantation Surgery, University Hospital Essen, Germany (Georgios C. Sotiropoulos) 


\section{Introduction}

The vast majority of injuries of the extrahepatic biliary system are iatrogenic, occurring most frequently during laparoscopic (LC) or open cholecystectomy (OC) [1,2]. The exact incidence of common bile duct injury (BDI) postcholecystectomy remains elusive, yet the currently available data addressing BDI during OC show that the incidence is relatively low, approximately $0.1-0.2 \%[2,3]$. Specifically with regard to BDI during $\mathrm{LC}$, the incidence of injuries as evidenced by national databases ranges from $0.1-0.55 \%$ (for major traumas), while the incidence of microbubbles with bile leakage is approximately $0.3 \%$, resulting in a total of $0.85 \%$ [2]. In a series of $12,397 \mathrm{LCs}$, Scott et al reported severe BDI in $0.3 \%$, minor injuries in $0.1 \%$ and cholorrhea in $0.4 \%$ of patients, with a total morbidity of $4 \%$ and mortality of $0.08 \%$ [ 4 ].

A plethora of factors associated with BDI have been identified; a surgical technique with insufficient exposure and failure to identify structures before ligation and division seem to be the most significant causes of major injuries $[5,6]$. In an analysis of 252 laparoscopic BDI, the authors showed that the main cause of the error was a visual misinterpretation in $97 \%$ of the cases, while technical errors accounted for only 3\% [7]. Moreover, while in the past BDI were considered more likely to occur in patients suffering from pancreatitis, cholangitis or acute cholecystitis, recent studies have shown that approximately $70 \%$ of BDI occur in cases where the only indication for cholecystectomy is symptomatic gallstones. [6]. The clinical course of patients with BDI would ideally be less complicated if they were promptly recognized at the time of surgery, but unfortunately this occurs only in about $25 \%$ of cases $[6,8]$. Moreover, in many patients there is a delayed referral to a specialized hepatobiliary (HPB) surgery center, despite actual signs of injury $[9,10]$. Management of patients with BDI post cholecystectomy is critical, whilst inadequate management of complex BDI with concurrent vascular injury has been associated with increased morbidity and mortality [11,12]. Early complications may include biliary peritonitis, systemic sepsis and multiple organ failure, and patients may later progress into end-stage liver disease (ESLD) [13,14].

In that setting, liver transplantation (LT) has been implemented as a last-resort strategy in a limited number of patients with ESLD secondary to usually multiple failed attempts to manage their BDI $[8,15,16]$. However, the outcomes in these patients remain ill-determined, mainly because of the small number of cases reported worldwide. To that end, the objective of our multicenter study was to identify and evaluate factors that cause or contribute to the progression to severe liver disease after BDI, ultimately requiring LT, and to assess the outcomes of patients transplanted for this indication.

\section{Patients and methods}

Fifteen LT centers were contacted and asked to provide information about patients who underwent LT after suffering from BDI during cholecystectomy. A questionnaire containing information regarding the following parameters was sent to all contributing centers: age, sex, the indication for cholecystectomy (cholecystitis vs. cholelithiasis), the type of approach for cholecystectomy (LC vs. OC), whether cholecystectomy was carried out in urgent or elective settings, whether BDI was recognized intraoperatively or postoperatively, the manifestation of BDI (biliary fistula or cholestasis), whether there was a concomitant vascular injury during cholecystectomy, the type of BDI according to Strasberg's classification [17], the treatment of BDI before LT (liver resection, bilioenteric anastomosis or repair through percutaneous transhepatic cholangiography [PTC] or endoscopic retrograde cholangiopancreatography [ERCP]), the time interval between BDI and its treatment before LT, the time interval between BDI and LT, the main indication for LT (liver failure vs. recurrent cholangitis), and morbidity, mortality, and survival after LT.

\section{Statistical analysis}

Data were expressed as median (min-max) for quantitative data and as absolute numbers and percentages for qualitative data. Survival times were expressed as median with standard error (SE) and 95\% confidence intervals (CI). Categorical variables were compared using the chi-square and Fisher's exact test. Logistic regression with the forward conditional method was used for multivariate analysis when the dependent outcome was a binary variable. Kaplan-Meier curves were used for the assessment of the time interval between bile duct injury and LT, as well as the assessment of survival after LT, while comparisons of survival among different groups were made using the log-rank test. Cox regression with the forward conditional method was used for multivariate survival analysis. All the tests were 2-tailed. Results were considered statistically significant if the P-value was less than 0.05. Statistical analysis was performed using the 25th edition of Statistical Package for Social Sciences (SPSS) (IBM Corporation, Armonk, NY, USA).

\section{Results}

\section{Patient characteristics}

Eight centers provided data for 34 patients (16 men and 18 women) who underwent LT as a last-resort treatment after BDI during cholecystectomy and were included in our study. Thirty of them (88.2\%) were referred for LT because of liver failure and the remaining $4(11.8 \%)$ because of recurrent cholangitis. The median age was 45 years (min-max: 22-69). Median follow-up period post LT was 57 months (min-max: 0 -158). Patient characteristics are shown in Table 1. 
Table 1 Patient characteristics

\begin{tabular}{|c|c|}
\hline Characteristics & Value \\
\hline Sex & Male: 16, Female: 18 \\
\hline Age & Median (min-max): 45 years (22-69) \\
\hline Acute cholecystitis & Yes: 17, No: 9, N/A: 8 \\
\hline Type of cholecystectomy & Laparoscopic: 14, Open: 20 \\
\hline $\begin{array}{l}\text { Setting of } \\
\text { cholecystectomy }\end{array}$ & Elective: 18, Urgent: 6, N/A: 10 \\
\hline Recognition of BDI & $\begin{array}{l}\text { Intraoperative: } 8 \text {, Postoperative: } 15 \text {, } \\
\text { N/A: } 11\end{array}$ \\
\hline Manifestation of BDI & Cholorrhea: 14, Cholestasis: 12, N/A: 8 \\
\hline $\begin{array}{l}\text { Concomitant vascular } \\
\text { injury }\end{array}$ & Yes: 8 , No: 26 \\
\hline Strasberg's classification & E2: 7, E3: 8, E4: 6, N/A: 13 \\
\hline Treatment of BDI & $\begin{array}{l}\text { ERCP: 6, PTC: } 2 \text {, Bilioenteric } \\
\text { anastomosis: } 21 \text {, liver resection: 1, N/A: } 4\end{array}$ \\
\hline $\begin{array}{l}\text { Time between BDI and } \\
\text { primary treatment }\end{array}$ & Median (min-max): 2 months (0-97) \\
\hline Indication for LT & Liver failure: 30, Recurrent cholangitis: 4 \\
\hline $\begin{array}{l}\text { Time between BDI and } \\
\text { LT }\end{array}$ & Median (min-max): 63 months $(0-336)$ \\
\hline $\begin{array}{l}\text { Postoperative } \\
\text { complications }\end{array}$ & $23(67.6 \%)$ \\
\hline $\begin{array}{l}\text { Major complications } \\
\text { (Clavien-Dindo } \geq 3 \mathrm{~b} \text { ) }\end{array}$ & $12(35.3 \%)$ \\
\hline 30-day mortality & $6(17.6 \%)$ \\
\hline Overall mortality & $10(29.4 \%)$ \\
\hline
\end{tabular}

\section{Time between BDI and LT}

The median time interval between BDI and LT was 63 months (min-max: 0-336). Patients who suffered BDI during LC required LT earlier (median time 19 months, SE 11.2, 95\%CI 0-41) than patients who suffered BDI during OC (median time 113 months, SE 27.6, 95\%CI 58.9-167.1) $(\mathrm{P}<0.001)$ (Fig. 1A). Furthermore, the time between BDI and LT was shorter if there was a concomitant vascular injury at the time of cholecystectomy (median time 12 months, SE 12.7, 95\%CI 0-36.9) than if there was not (median time 75 months, SE 28.2, 95\%CI 19.8-130.2) $(\mathrm{P}<0.001)$ (Fig. 1B). In addition, there was an association between the type of BDI according to the Strasberg classification and the time period until LT. In particular, the median time interval between BDI and LT was 46 months for E2 injuries (SE 22.3, 95\%CI 2.4-89.6), 113 months for E3 injuries (SE 43.8, 95\%CI 27.1-198.9) and 19 months for E4 injuries (SE 11, 95\%CI 0-40.6) ( $\mathrm{P}=0.007)$ (Fig. 1C). However, Cox regression analysis revealed that the only independent risk factor associated with a shorter time interval between BDI and LT was the presence of a concomitant vascular injury (hazard ratio 10.69, 95\%CI 1.134-100.8, $\mathrm{P}=0.039$ ). Results concerning time until LT are listed in Table 2.
Table 2 Time between BDI and LT

\begin{tabular}{|c|c|c|}
\hline Parameter & $\begin{array}{l}\text { Univariate analysis } \\
\text { (median times) }\end{array}$ & $\begin{array}{l}\text { Multivariate } \\
\text { analysis }\end{array}$ \\
\hline Sex & $\begin{array}{l}\text { Male: } 52 \text { months } \\
\text { Female: } 75 \text { months } \\
\mathrm{P}=0.314\end{array}$ & $\mathrm{P}=0.318$ \\
\hline Age (years) & $\begin{array}{l}\leq 50: 67 \text { months } \\
>50: 44 \text { months } \\
\mathrm{P}=0.226\end{array}$ & $\mathrm{P}=0.509$ \\
\hline Acute cholecystitis & $\begin{array}{l}\text { No: } 19 \text { months } \\
\text { Yes: } 58 \text { months } \\
\mathrm{P}=0.267\end{array}$ & $\mathrm{P}=0.695$ \\
\hline $\begin{array}{l}\text { Type of } \\
\text { cholecystectomy }\end{array}$ & $\begin{array}{l}\text { Open: } 113 \text { months } \\
\text { Laparoscopic: } 19 \text { months } \\
\mathrm{P}<0.001\end{array}$ & $\mathrm{P}=0.35$ \\
\hline $\begin{array}{l}\text { Setting of } \\
\text { cholecystectomy }\end{array}$ & $\begin{array}{l}\text { Elective: } 58 \text { months } \\
\text { Urgent: } 12 \text { months } \\
\mathrm{P}=0.214\end{array}$ & $\mathrm{P}=0.078$ \\
\hline $\begin{array}{l}\text { Recognition of } \\
\text { BDI }\end{array}$ & $\begin{array}{l}\text { Intraoperative: } 29 \text { months } \\
\text { Postoperative: } 51 \text { months } \\
\mathrm{P}=0.827\end{array}$ & $\mathrm{P}=0.927$ \\
\hline $\begin{array}{l}\text { Manifestation of } \\
\text { BDI }\end{array}$ & $\begin{array}{l}\text { Cholorrhea: } 58 \text { months } \\
\text { Cholestasis: } 52 \text { months } \\
\mathrm{P}=0.266\end{array}$ & $\mathrm{P}=0.734$ \\
\hline $\begin{array}{l}\text { Concomitant } \\
\text { vascular injury }\end{array}$ & $\begin{array}{l}\text { No: } 75 \text { months } \\
\text { Yes: } 12 \text { months } \\
\mathrm{P}<0.001\end{array}$ & $\begin{array}{c}\text { HR } 10.69 \\
95 \% \text { CI } 1.134- \\
100.8 \\
\mathrm{P}=0.039\end{array}$ \\
\hline $\begin{array}{l}\text { Strasberg's } \\
\text { classification }\end{array}$ & $\begin{array}{l}\text { E2: } 46 \text { months } \\
\text { E3: } 113 \text { months } \\
\text { E4: } 19 \text { months } \\
P=0.007\end{array}$ & $\mathrm{P}=0.264$ \\
\hline Treatment of BDI & $\begin{array}{l}\text { PTC/ERCP: } 51 \text { months } \\
\text { Bilioenteric anastomosis: } 108 \\
\text { months } \\
\mathrm{P}=0.935\end{array}$ & $\mathrm{P}=0.337$ \\
\hline $\begin{array}{l}\text { Time between BDI } \\
\text { and treatment }\end{array}$ & $\begin{array}{l}\leq 1 \text { month: } 44 \text { months } \\
>1 \text { month: } 52 \text { months } \\
\mathrm{P}=0.341\end{array}$ & $\mathrm{P}=0.933$ \\
\hline Indication for LT & $\begin{array}{l}\text { Recurrent cholangitis: } 25 \\
\text { months } \\
\text { Liver failure: } 59 \text { months } \\
P=0.604\end{array}$ & $\mathrm{P}=0.902$ \\
\hline
\end{tabular}

BDI, bile duct injury; $L T$, liver transplantation; $P T C$, percutaneous transhepatic cholangiography; ERCP, endoscopic retrograde cholangiopancreatography; HR, hazard ratio; CI, confidence interval

\section{Postoperative outcomes after LT}

Twenty-three patients (67.6\%) had postoperative complications following LT. Patients who suffered BDI during OC had higher postoperative morbidity rates $(17 / 20 ; 85 \%)$ than patients who suffered BDI during LC $(6 / 14 ; 42.9 \%)$ $(\mathrm{P}=0.023)$. Moreover, patients who underwent LT more than 2 years after BDI were more likely to experience postoperative complications $(19 / 25 ; 76 \%)$ than patients who underwent LT within 2 years after BDI $(2 / 7 ; 28.6 \%)(\mathrm{P}=0.032)$. Nevertheless, 

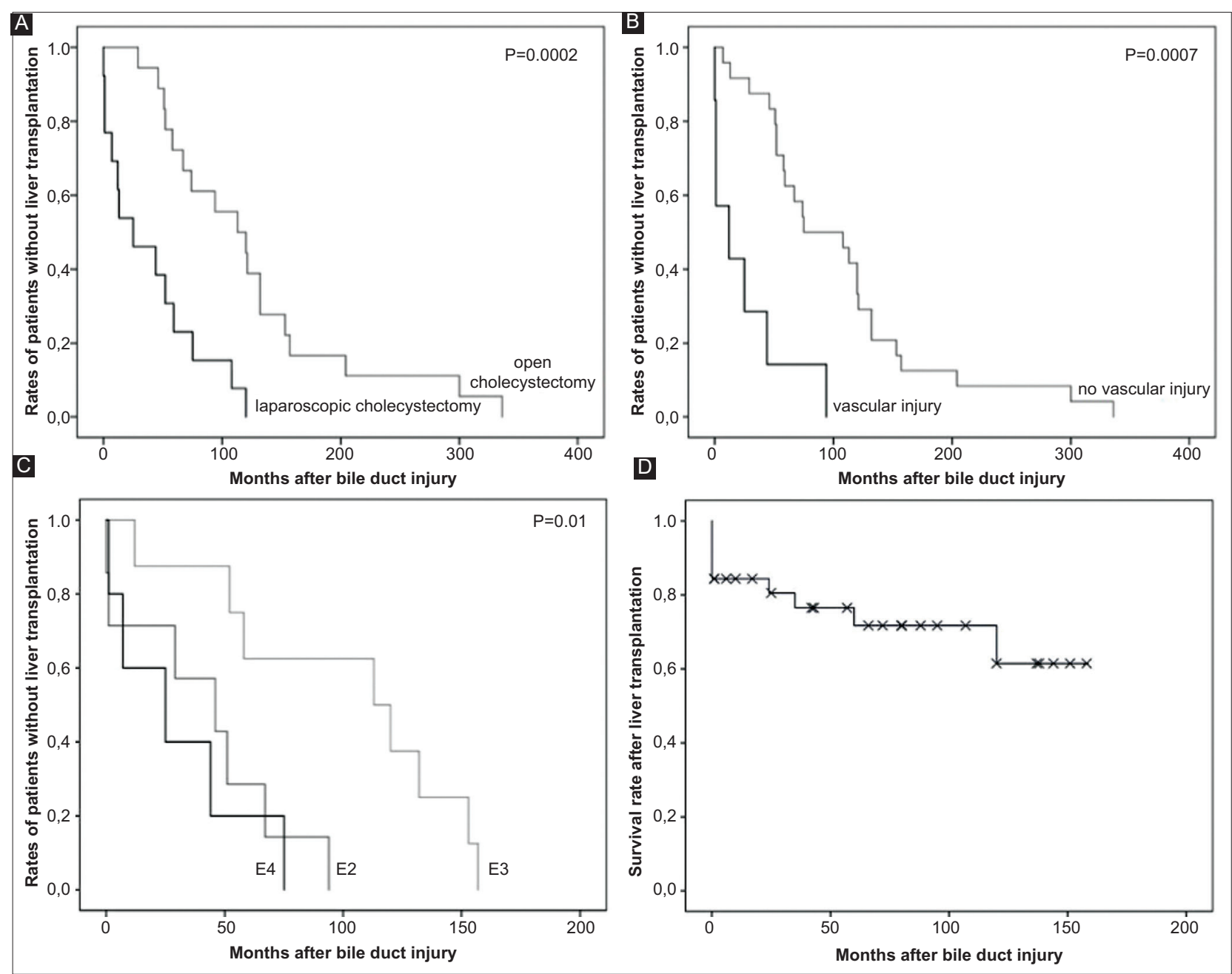

Figure 1 (A) Time between BDI and LT according to type of cholecystectomy; (B) Time between BDI and LT according to vascular injury; (C) Time between BDI and LT according to type of bile duct injury; (D) Overall survival after LT $B D I$, bile duct injury; LT, liver transplantation

logistic regression analysis showed no independent risk factor for postoperative morbidity after LT. Twelve patients $(35.3 \%)$ had major complications (Clavien-Dindo classification [18] 3b or higher) after LT. Patients older than 50 years had lower rates of major complications post LT $(2 / 15 ; 13.3 \%)$ when compared with younger patients $(10 / 19 ; 52.6 \%)(\mathrm{P}=0.017)$. Nonetheless, logistic regression analysis revealed no independent risk factor for major complications post LT.

Six patients $(17.6 \%)$ died within the first month post LT. However, no association was found between postoperative mortality and any of the tested parameters in either unior multivariate analysis. Results regarding postoperative outcomes after LT are shown in Table 3.

\section{Overall survival after LT}

The median surveillance period after LT was 57 months (minmax: 0-158). Ten patients (29.4\%) died during the surveillance period after LT. Overall survival rates were $82.4 \%(28 / 34)$ at
1 year, $76.5 \%$ at 3 years (26/34), $73.5 \%$ at 5 years (25/34), and $70.6 \%$ at 10 years (24/34) after LT (Fig. 1D). Patients older than 50 years old had longer overall survival post LT (median survival not reached yet) than younger patients (median survival 60 months, SE 18.7, 95\%CI 48.2-121.6) $(\mathrm{P}=0.041)$. Furthermore, patients who received treatment for BDI within the first month (bilioenteric anastomosis or treatment through PTC or ERCP) had shorter overall survival after LT (median survival 35 months, SE 45.8, 95\%CI 0-124.8) than patients who received treatment more than 1 month after BDI (median survival not reached yet) $(\mathrm{P}=0.026)$. However, Cox regression analysis did not reveal any independent prognostic factor of overall survival after LT. Results concerning overall survival post LT are listed in Table 4.

\section{Discussion}

LT for patients who suffered from BDI during cholecystectomy was first reported almost 30 years ago [19], 
Table 3 Postoperative outcomes after LT

\begin{tabular}{|c|c|c|c|c|c|c|}
\hline \multirow[t]{2}{*}{ Parameter } & \multicolumn{2}{|c|}{ Complications } & \multicolumn{2}{|c|}{ Major complications } & \multicolumn{2}{|c|}{ Mortality } \\
\hline & $\begin{array}{l}\text { Univariate } \\
\text { analysis }\end{array}$ & $\begin{array}{l}\text { Multivariate } \\
\text { analysis }\end{array}$ & $\begin{array}{l}\text { Univariate } \\
\text { analysis }\end{array}$ & $\begin{array}{c}\text { Multivariate } \\
\text { analysis }\end{array}$ & $\begin{array}{c}\text { Univariate } \\
\text { analysis }\end{array}$ & $\begin{array}{c}\text { Multivariate } \\
\text { analysis }\end{array}$ \\
\hline \multicolumn{7}{|l|}{ Sex } \\
\hline Male & $11 / 16$ & & $7 / 16$ & & $3 / 16$ & \\
\hline Female & $12 / 18$ & & $5 / 18$ & & $3 / 16$ & \\
\hline P-value & 0.897 & $>0.99$ & 0.331 & 0.303 & $>0.99$ & 0.389 \\
\hline \multicolumn{7}{|l|}{ Age (years) } \\
\hline$\leq 50$ & $15 / 19$ & & $10 / 19$ & & $5 / 19$ & \\
\hline$>50$ & $8 / 15$ & & $2 / 15$ & & $1 / 15$ & \\
\hline P-value & 0.151 & 0.14 & 0.017 & 0.1 & 0.196 & 0.107 \\
\hline \multicolumn{7}{|l|}{ Acute cholecystitis } \\
\hline No & $5 / 9$ & & $3 / 9$ & & $2 / 9$ & \\
\hline Yes & $11 / 17$ & & $4 / 17$ & & $1 / 17$ & \\
\hline P-value & 0.692 & 0.121 & 0.661 & 0.165 & 0.268 & $>0.99$ \\
\hline \multicolumn{7}{|l|}{ Type of cholecystectomy } \\
\hline Open & $17 / 20$ & & $8 / 20$ & & $4 / 20$ & \\
\hline Laparoscopic & $6 / 14$ & & $4 / 14$ & & $2 / 14$ & \\
\hline P-value & 0.023 & $>0.99$ & 0.717 & 0.819 & $>0.99$ & 0.292 \\
\hline \multicolumn{7}{|l|}{ Setting of cholecystectomy } \\
\hline Elective & $11 / 18$ & & $4 / 18$ & & $2 / 18$ & \\
\hline Urgent & $4 / 6$ & & $3 / 6$ & & $1 / 6$ & \\
\hline P-value & $>0.99$ & 0.439 & 0.307 & 0.898 & $>0.99$ & 0.49 \\
\hline \multicolumn{7}{|l|}{ Recognition of BDI } \\
\hline Intraoperative & $5 / 8$ & & $2 / 8$ & & $2 / 8$ & \\
\hline Postoperative & $9 / 15$ & & $4 / 15$ & & $1 / 15$ & \\
\hline P-value & $>0.99$ & 0.54 & $>0.99$ & 0.477 & 0.269 & 0.107 \\
\hline \multicolumn{7}{|l|}{ Manifestation of BDI } \\
\hline Cholorrhea & $11 / 14$ & & $7 / 14$ & & $4 / 14$ & \\
\hline Cholestasis & $6 / 12$ & & $3 / 12$ & & $0 / 12$ & \\
\hline P-value & 0.218 & 0.54 & 0.248 & 0.554 & 0.1 & 0.389 \\
\hline \multicolumn{7}{|l|}{ Concomitant vascular injury } \\
\hline No & $20 / 26$ & & $9 / 26$ & & $4 / 26$ & \\
\hline Yes & $3 / 8$ & & $3 / 8$ & & $2 / 8$ & \\
\hline P-value & 0.079 & 0.221 & $>0.99$ & 0.477 & 0.609 & 0.49 \\
\hline \multicolumn{7}{|l|}{ Strasberg's classification } \\
\hline E2 & $5 / 7$ & & $3 / 7$ & & $1 / 7$ & \\
\hline E3 & $5 / 8$ & & $1 / 8$ & & $1 / 8$ & \\
\hline E4 & $3 / 6$ & & $2 / 6$ & & $1 / 6$ & \\
\hline P-value & 0.856 & 0.472 & 0.491 & 0.588 & $>0.99$ & 0.574 \\
\hline \multicolumn{7}{|l|}{ Treatment of BDI } \\
\hline PTC/ERCP & $6 / 8$ & & $4 / 8$ & & $0 / 8$ & \\
\hline Bilioenteric anastomosis & $15 / 21$ & & $7 / 21$ & & $5 / 21$ & \\
\hline
\end{tabular}

(Contd...) 
Table 3 (Continued)

\begin{tabular}{|c|c|c|c|c|c|c|}
\hline \multirow[t]{2}{*}{ Parameter } & \multicolumn{2}{|c|}{ Complications } & \multicolumn{2}{|c|}{ Major complications } & \multicolumn{2}{|c|}{ Mortality } \\
\hline & $\begin{array}{l}\text { Univariate } \\
\text { analysis }\end{array}$ & $\begin{array}{l}\text { Multivariate } \\
\text { analysis }\end{array}$ & $\begin{array}{l}\text { Univariate } \\
\text { analysis }\end{array}$ & $\begin{array}{c}\text { Multivariate } \\
\text { analysis }\end{array}$ & $\begin{array}{c}\text { Univariate } \\
\text { analysis }\end{array}$ & $\begin{array}{c}\text { Multivariate } \\
\text { analysis }\end{array}$ \\
\hline P-value & $>0.99$ & 0.54 & 0.433 & 0.819 & 0.283 & 0.292 \\
\hline \multicolumn{7}{|c|}{ Time between BDI and treatment } \\
\hline$\leq 1$ month & $5 / 7$ & & $4 / 7$ & & $3 / 7$ & \\
\hline$>1$ month & $8 / 10$ & & $4 / 10$ & & $1 / 10$ & \\
\hline P-value & $>0.99$ & $>0.99$ & 0.637 & 0.819 & 0.25 & 0.197 \\
\hline \multicolumn{7}{|l|}{ Indication for LT } \\
\hline Recurrent cholangitis & $3 / 4$ & & $2 / 4$ & & $0 / 4$ & \\
\hline Liver failure & $20 / 30$ & & $10 / 30$ & & $6 / 30$ & \\
\hline P-value & $>0.99$ & 0.121 & 0.602 & 0.165 & $>0.99$ & 0.725 \\
\hline \multicolumn{7}{|c|}{ Time between BDI and LT } \\
\hline$\leq 2$ years & $2 / 7$ & & $2 / 7$ & & $2 / 7$ & \\
\hline$>2$ years & $19 / 25$ & & $9 / 25$ & & $3 / 25$ & \\
\hline P-value & 0.032 & $>0.99$ & $>0.99$ & 0.898 & 0.296 & 0.598 \\
\hline
\end{tabular}

but outcomes at that time were dismal and long-term results disappointing. Reports from the 1970s showed that approximately $8 \%$ of patients with BDI ultimately suffered from biliary cirrhosis due to failed attempts to manage biliary outflow obstruction, with associated increased mortality. At the time, LT was not a pursuable option [20]. More recently published series have shown that approximately 4-19\% of BDI patients are eligible for LT, mainly due to secondary biliary cirrhosis (SBC), with acceptable reported posttransplant long-term outcomes $[8,21,22]$. Nonetheless, it should be taken into account that the exact denominator of patients with BDI might be higher than reported; thus, the exact proportion of patients led to LT remains poorly determined.

Whilst concomitant major vascular injuries may lead to acute liver failure, long-term damage to the liver parenchyma is difficult to predict in patients with BDI. Vascular injuries or unresolved partial biliary obstruction may result in acute liver failure, liver atrophy, chronic intrahepatic infection, abscess formation or SBC. Consequently, careful evaluation is mandated in these patients. Liver resection has been advocated in a selected number of patients with BDI, the majority being patients with continuous sepsis in obstructed segments or those whose drainage of extrahepatic bile ducts is not adequate because of conduit or fibrosis damage $[23,24]$.

According to a recent review of 1756 BDIs following cholecystectomy, 99 patients $(5.6 \%)$ were reported to require hepatectomy, 16 of whom had concomitant arterial injury [24]. Occasionally, early liver resection is required for combined arterial injury, portal vein injury and biliary trauma, yet the outcomes are poor [25]. The right lobe is usually affected by sepsis and atrophy, as is the right hepatic duct, because the right artery is more likely to be damaged during cholecystectomy [25]. In our series, no patient initially underwent liver resection, possibly highlighting that it may represent a more effective approach in treating these patients and preventing them from requiring LT.

Currently available data on patients undergoing LT after BDI following cholecystectomy are scanty and mainly derive from case reports and small case series [15,23,26-28]. Ardiles et al conducted a retrospective multicenter national survey in Argentina, aiming to collect clinical data on patients referred for LT as a consequence of iatrogenic BDI during a 14-year period [8]; 16 patients (6 LC and $10 \mathrm{OC}$ ) were led to LT, after a mean interval of 71 months from BDI occurrence during cholecystectomy, with the most common indication being SBC (93.7\%). Authors observed that the percentage of LT performed for BDI declined over the study period. The reported 5- and 10-year survival rates were $68 \%$ and $45 \%$, respectively [8]. In the largest published series, from the Spanish Liver Transplantation Study Groups, during a 24-year period 24 patients underwent LT because of BDI following cholecystectomy [16]. The authors reported that 12 and 13 patients initially suffered from BDI after LC and OC, respectively, whereas the median time from index surgery to LT was 3 years, significantly lower than the respective interval of 5.25 years in our series. Similarly to the previous mentioned and our study, the most common indication leading to LT was SBC (74\% of patients). Seven patients underwent emergent LT for acute liver failure after BDI associated with a vascular injury, with only 1 survivor. In the same study, associated vascular injuries were more commonly encountered in patients who had initially undergone LC, similarly to our study where $87.5 \%$ of concomitant vascular injuries were sustained during a laparoscopic approach. Overall post-LT mortality was $42 \%$ 
Table 4 Overall survival after LT

\begin{tabular}{|c|c|c|}
\hline Parameter & $\begin{array}{l}\text { Univariate analysis } \\
\text { (median times) }\end{array}$ & $\begin{array}{l}\text { Multivariate } \\
\text { analysis }\end{array}$ \\
\hline Sex & $\begin{array}{l}\text { Male: not reached yet } \\
\text { Female: not reached yet } \\
\mathrm{P}=0.386\end{array}$ & $\mathrm{P}=0.592$ \\
\hline Age (years) & $\begin{array}{l}\leq 50: 60 \text { months } \\
>50: \text { not reached yet } \\
\mathrm{P}=0.041\end{array}$ & $\mathrm{P}=0.475$ \\
\hline $\begin{array}{l}\text { Acute } \\
\text { cholecystitis }\end{array}$ & $\begin{array}{l}\text { No: not reached yet } \\
\text { Yes: not reached yet } \\
\mathrm{P}=0.565\end{array}$ & $\mathrm{P}=0.103$ \\
\hline $\begin{array}{l}\text { Type of } \\
\text { cholecystectomy }\end{array}$ & $\begin{array}{l}\text { Open: not reached yet } \\
\text { Laparoscopic: not reached yet } \\
\mathrm{P}=0.306\end{array}$ & $\mathrm{P}=0.705$ \\
\hline $\begin{array}{l}\text { Setting of } \\
\text { cholecystectomy }\end{array}$ & $\begin{array}{l}\text { Elective: not reached yet } \\
\text { Urgent: not reached yet } \\
\mathrm{P}=0.827\end{array}$ & $\mathrm{P}=0.238$ \\
\hline $\begin{array}{l}\text { Recognition of } \\
\text { BDI }\end{array}$ & $\begin{array}{l}\text { Intraoperative: not reached yet } \\
\text { Postoperative: not reached yet } \\
\mathrm{P}=0.421\end{array}$ & $\mathrm{P}=0.475$ \\
\hline $\begin{array}{l}\text { Manifestation of } \\
\text { BDI }\end{array}$ & $\begin{array}{l}\text { Cholorrhea: not reached yet } \\
\text { Cholestasis: not reached yet } \\
\mathrm{P}=0.188\end{array}$ & $\mathrm{P}=0.886$ \\
\hline $\begin{array}{l}\text { Concomitant } \\
\text { vascular injury }\end{array}$ & $\begin{array}{l}\text { No: not reached yet } \\
\text { Yes: } 120 \text { months } \\
P=0.709\end{array}$ & $\mathrm{P}=0.768$ \\
\hline $\begin{array}{l}\text { Strasberg's } \\
\text { classification }\end{array}$ & $\begin{array}{l}\text { E2: not reached yet } \\
\text { E3: not reached yet } \\
\text { E4: not reached yet } \\
P=0.996\end{array}$ & $\mathrm{P}=0.746$ \\
\hline Treatment of BDI & $\begin{array}{l}\text { PTC/ERCP: not reached yet } \\
\text { Bilioenteric anastomosis: not } \\
\text { reached yet } \\
\mathrm{P}=0.23\end{array}$ & $\mathrm{P}=0.157$ \\
\hline $\begin{array}{l}\text { Time between } \\
\text { BDI and } \\
\text { treatment }\end{array}$ & $\begin{array}{l}\leq 1 \text { month: } 35 \text { months } \\
>1 \text { month: not reached yet } \\
\mathrm{P}=0.026\end{array}$ & $\mathrm{P}=0.116$ \\
\hline Indication for LT & $\begin{array}{l}\text { Recurrent cholangitis: not } \\
\text { reached yet } \\
\text { Liver failure: not reached yet } \\
\mathrm{P}=0.253\end{array}$ & $\mathrm{P}=0.739$ \\
\hline $\begin{array}{l}\text { Time between } \\
\mathrm{BDI} \text { and LT }\end{array}$ & $\begin{array}{l}\leq 2 \text { years: not reached yet } \\
>2 \text { years: not reached yet } \\
\mathrm{P}=0.795\end{array}$ & $\mathrm{P}=0.274$ \\
\hline
\end{tabular}

and $7.7 \%$ for LC and OC patients, respectively, with a 5-year survival of $68 \%$ [16].

Inferior outcomes in these patients are at least partially due to the elevated degree of difficulty in performing LT in these patients, which can be particularly challenging. They are highly likely to have undergone a significant number of endoscopic or/and surgical procedures and therefore their anatomy, both biliary and vascular, is far from normal [15,29]. Additionally, the presence of multiple adhesions formed as a result of severe inflammation and/or multiple surgical interventions can be very difficult to manage [30]. Notably, 3 of the patients included in our analysis had 2 or more redo hepaticojejunostomy anastomoses. In the first published series of 4 patients who underwent LT for BDI, because of a combination of advanced cirrhosis, severe portal hypertension and dense abdominal adhesions, none of the patients survived the procedure [19]. When comparing patients who underwent LT for ESLD secondary to BDI during cholecystectomy with patients who underwent LT for other indications during the same study period, Santibanes et al showed that, although morbidity and long-term survival were similar in the 2 groups, the former group had significantly more protracted operative times, were more challenging and had higher blood transfusion requirements [30]. Based on our analysis, patients who underwent LT more than 2 years after the BDI were more likely to experience postoperative complications, possibly indicating the presence of tenacious adhesions compromising hilar dissection, poorer performance status, recurrent cholangitis and potential sepsis, as well as ischemic damage with vanishing ducts, compared to those who underwent LT within 2 years after the BDI.

To the best of our knowledge, our study presents the largest reported cohort of patients who underwent LT as a last resort because of BDI during cholecystectomy. Nonetheless, several limitations must be taken into account before solid conclusions can be drawn. First, the retrospective nature of our study and the small number of patients included constitute significant limitations. Since these patients underwent LT in a specialized center other than the one in which the original BDI occurred, it was not possible to evaluate confounding factors such as surgeon's experience and surgical expertise, which could correlate with the complexity of the injury. Collecting accurate data for such patients was very challenging; the majority follow a long and transient course from cholecystectomy to LT, which may involve admissions and interventional procedures in more than 2 hospitals, or even in different countries, which may not be reported adequately. Additionally, the indications and criteria for LT may vary between reference centers. The limited number of patients included made it harder to determine significant associations between predictors and analyzed outcomes.

Long-lasting unsuccessful management of BDI can be detrimental by making LT challenging, thus highlighting the need for these patients to be referred more promptly to specialized HPB/LT units. Despite the relatively high related morbidity and mortality, LT for patients with ESLD secondary to otherwise unmanageable BDI following cholecystectomy seems to confer acceptable long-term survival compared to other indications. However, this is a real scenario that represents one of the most dreadful outcomes of a supposedly straightforward surgical procedure such as cholecystectomy. 


\section{Summary Box}

\section{What is already known:}

- The vast majority of extrahepatic biliary system injuries are iatrogenic, occurring most frequently during laparoscopic (LC) or open cholecystectomy (OC)

- Long-term damage to the liver parenchyma is difficult to predict in patients with poorly managed BDI

- Liver transplantation (LT) has been used as a last resort in patients with end-stage liver disease due to bile duct injuries (BDI) following cholecystectomy

\section{What the new findings are:}

- Overall survival rates after LT were $82.4 \%$ at 1 year, $76.5 \%$ at 3 years, $73.5 \%$ at 5 years and $70.6 \%$ at 10 years

- The time interval between BDI and LT was shorter if there was a concomitant vascular injury at the time of cholecystectomy

- Despite the relatively high related morbidity and mortality, LT for patients with end-stage liver disease secondary to otherwise unmanageable BDI following cholecystectomy seems to confer acceptable longterm survival compared to other indications

\section{References}

1. Archer SB, Brown DW, Smith CD, Branum GD, Hunter JG. Bile duct injury during laparoscopic cholecystectomy: results of a national survey. Ann Surg 2001;234:549-558.

2. Waage A, Nilsson M. Iatrogenic bile duct injury: a populationbased study of 152776 cholecystectomies in the Swedish Inpatient Registry. Arch Surg 2006;141:1207-1213.

3. Richardson MC, Bell G, Fullarton GM. Incidence and nature of bile duct injuries following laparoscopic cholecystectomy: an audit of 5913 cases. West of Scotland Laparoscopic Cholecystectomy Audit Group. Br J Surg 1996;83:1356-1360.

4. Scott TR, Zucker KA, Bailey RW. Laparoscopic cholecystectomy: a review of 12,397 patients. Surg Laparosc Endosc 1992;2:191-198.

5. Fletcher DR, Hobbs MS, Tan P, et al. Complications of cholecystectomy: risks of the laparoscopic approach and protective effects of operative cholangiography: a population-based study. Ann Surg 1999;229:449-457.

6. Thomson BN, Cullinan MJ, Banting SW, Collier NA; Universities of Melbourne Hepatobiliary Group. Recognition and management of biliary complications after laparoscopic cholecystectomy. ANZ J Surg 2003;73:183-188.

7. Way LW, Stewart L, Gantert W, et al. Causes and prevention of laparoscopic bile duct injuries: analysis of 252 cases from a human factors and cognitive psychology perspective. Ann Surg 2003;237:460-469.

8. Ardiles V, McCormack L, Quinonez E, et al. Experience using liver transplantation for the treatment of severe bile duct injuries over 20 years in Argentina: results from a National Survey. $H P B$ (Oxford) 2011;13:544-550.
9. Mirza DF, Narsimhan KL, Ferraz Neto BH, Mayer AD, McMaster P, Buckels JA. Bile duct injury following laparoscopic cholecystectomy: referral pattern and management. Br J Surg 1997;84:786-790.

10. Martinez-Lopez S, Upasani V, Pandanaboyana S, et al. Delayed referral to specialist centre increases morbidity in patients with bile duct injury (BDI) after laparoscopic cholecystectomy (LC). Int J Surg 2017;44:82-86.

11. Stewart L. Iatrogenic biliary injuries: identification, classification, and management. Surg Clin North Am 2014;94:297-310.

12. Pesce A, Palmucci S, La Greca G, Puleo S. Iatrogenic bile duct injury: impact and management challenges. Clin Exp Gastroenterol 2019;12:121-128.

13. Mercado MA, Dominguez I. Classification and management of bile duct injuries. World J Gastrointest Surg 2011;3:43-48.

14. Nordin A, Halme L, Makisalo H, Isoniemi H, Hockerstedt K. Management and outcome of major bile duct injuries after laparoscopic cholecystectomy: from therapeutic endoscopy to liver transplantation. Liver Transplant 2002;8:1036-1043.

15. de Santibanes E, Ardiles V, Gadano A, Palavecino M, Pekolj J, Ciardullo M. Liver transplantation: the last measure in the treatment of bile duct injuries. World J Surg 2008;32:1714-1721.

16. Parrilla P, Robles R, Varo E, et al. Liver transplantation for bile duct injury after open and laparoscopic cholecystectomy. Br J Surg 2014;101:63-68.

17. Strasberg SM, Hertl M, Soper NJ. An analysis of the problem of biliary injury during laparoscopic cholecystectomy. J Am Coll Surg 1995;180:101-125.

18. Clavien PA, Barkun J, de Oliveira ML, et al. The Clavien-Dindo classification of surgical complications: five-year experience. Ann Surg 2009;250:187-196.

19. Moossa AR, Mayer AD, Stabile B. Iatrogenic injury to the bile duct. Who, how, where? Arch Surg 1990;125:1028-1030.

20. Braasch JW, Bolton JS, Rossi RL. A technique of biliary tract reconstruction with complete follow-up in 44 consecutive cases. Ann Surg 1981;194:635-638.

21. Schmidt SC, Langrehr JM, Hintze RE, Neuhaus P. Long-term results and risk factors influencing outcome of major bile duct injuries following cholecystectomy. Br J Surg 2005;92:76-82.

22. de Santibanes E, Palavecino M, Ardiles V, Pekolj J. Bile duct injuries: management of late complications. Surg Endosc 2006;20:1648-1653.

23. Thomson BN, Parks RW, Madhavan KK, Garden OJ. Liver resection and transplantation in the management of iatrogenic biliary injury. World J Surg 2007;31:2363-2369.

24. Truant S, Boleslawski E, Lebuffe G, Sergent G, Pruvot FR. Hepatic resection for post-cholecystectomy bile duct injuries: a literature review. HPB (Oxford) 2010;12:334-341.

25. Strasberg SM, Helton WS. An analytical review of vasculobiliary injury in laparoscopic and open cholecystectomy. HPB (Oxford) 2011;13:1-14.

26. Sotiropoulos GC, Tsaparas P, Kykalos S, Machairas N, Molmenti EP, Paul A. From laparoscopic cholecystectomy to liver transplantation: when the gallbladder becomes the Pandora s box. Chirurgia (Bucur) 2016;111:450-454.

27. Lubikowski J, Chmurowicz T, Post M, et al. Liver transplantation as an ultimate step in the management of iatrogenic bile duct injury complicated by secondary biliary cirrhosis. Ann Transplant 2012;17:38-44.

28. Yan JQ, Peng $\mathrm{CH}$, Shen BY, et al. Liver transplantation as a treatment for complicated bile duct injury. Hepatogastroenterology 2011;58:8-13.

29. Loinaz C, Gonzalez EM, Jimenez C, et al. Long-term biliary complications after liver surgery leading to liver transplantation. World J Surg 2001;25:1260-1263.

30. de Santibanes E, Pekolj J, McCormack L, et al. Liver transplantation for the sequelae of intra-operative bile duct injury. HPB (Oxford) 2002;4:111-115. 